\title{
Recent advances and near future of insulin production and therapy
}

\author{
Darian Allen', Cheng-Hai Ruan², Bridgette King ${ }^{1}$ \& Ke-He Ruan*,1 \\ ${ }^{1}$ Center for Experimental Therapeutics \& Pharmacoinformatics, \& Department of Pharmacological \& Pharmaceutical Sciences, \\ College of Pharmacy, University of Houston, Houston, TX 77004, USA \\ ${ }^{2}$ Texas Center of Lifestyle Medicine, Ruan Medical Group, 5252 Hollister, Suite 201, Houston, TX 77040, USA \\ *Author for correspondence: Tel.: +1 713743 1771; Fax: +1 713743 1884; khruan@uh.edu
"The high level of biologically active proinsulin being expressed in transgenic plants provides low-cost production of injectable and oral proinsulin delivery"

First draft submitted: 29 April 2019; Accepted for publication: 9 May 2019; Published online: 30 August 2019

Keywords: E. coli • insulin • insulin mutant • recombinant insulin • stem cells • yeast

The World Health Organization (WHO) has listed insulin as an 'essential medicine'; a basic medicine which "satisfies the priority healthcare needs of the population" [1]. This clearly indicates the significance of insulin for human disease treatment. With an increase in diabetic patients worldwide, novel methods for more effective insulin production and application are currently being pursued. Techniques to improve insulin drug design, biosynthesis and delivery are still hot topics for the fields of medicinal chemistry, pharmacology and pharmaceutics. This editorial will address the popular and interesting topic of the recent advances and future perspectives of insulin production and therapy. It should provide a valuable resource for researchers, and clinicians in the field of diabetes and insulin therapy.

\section{Insulin history, significance \& basic structure}

Zion Market Research has predicted a market for insulin to reach $\$ 43.6$ billion USD in 2021 [2]. Carbohydrate and fat metabolism is primarily regulated by insulin, making insulin an important and essential medication. Discovered in 1921 by Banting and Best as a polypeptide hormone, insulin is produced by the islets of Langerhans in the pancreas [3]. Insulin is synthesized by beta cells of the pancreas as a single chain of three peptides A, B and C; known as pre-proinsulin [4,5]. Pre-proinsulin is cleaved, and the polypeptide is translocated to the human endoplasmic reticulum forming proinsulin. Conversion to mature insulin is achieved by proteolytic enzymes known as prohormone convertases PC1/PC3 and PC2, cleaving at two positions to release the central C-peptide [6]. The resulting mature insulin is comprised of 51 amino acids ( 30 amino acid B chains and 21 amino acid A chains) linked together by two interchain disulfide bonds to form a monomer [7-10]. In addition, A-chain contains one intra-chain disulfide bond. Cleavage of C-terminal amino acid from either chain, and the two interchain disulfide bonds eliminate activity of the mature insulin.

\section{Advance in insulin mutants: structure $\&$ function relationship}

Insulin secretion in the body peaks $1 \mathrm{~h}$ after eating followed by its decline within the next $2 \mathrm{~h}$ in healthy individuals [3]. In contrast, diabetic patients require insulin to have a specific peak time, onset of action and duration of action to achieve a 24-h insulin profile and to avoid nocturnal hypoglycemia; thus, the need for multiple insulin formulations [3]. Once insulin has been injected, the molecule forms a hexamer held together by a single zinc ion [3]. The hexamers dissociate into dimers and monomers that diffuse and penetrate capillary walls to reach the bloodstream. The rate of the dissociation is manipulated in different formulations. Rapid acting insulin formulations are produced by recombinant DNA technology by switching single or two amino acid residues in the insulin molecule. The amino acid modification does not alter receptor binding, but inhibits formation of insulin dimers and hexamers [11,12]. Larger amounts of insulin monomers are readily made available for fast absorption. Current market examples include Lispro and Aspart. Lispros' analog has its ProB28 and LysB29 residues on the C-terminal end of the B-chain reversed to LysB28 and ProB29 [11]. Insulin Aspart has its proline at position 28 on 
the B-chain replaced by aspartic acid [12]. Aspart's modification yields an increase in charge repulsion to prevent hexamers from forming, creating a faster acting insulin. Intermediate analogs, NPH (Neutral- Protamine- Haledon) and LENTE, exhibit onset of action near 1-2 h, peak action 6-10 $\mathrm{h}$ and activity duration of 10-16 $\mathrm{h}$ [3]. Absorption rates are decreased in NPH due to the addition of protamine, a small arginine-rich nuclear protein, which slows down the onset and increases the duration of insulin action. LENTE achieves the same by the addition of zinc to its formulation.

\section{Advance in recombinant insulin production}

Recombinant human insulin production is primarily produced in E. coli or Saccharomyces cerevisiae expression systems [13]. Initially, E. coli was the preferred expression system for large-scale recombinant insulin production due to its high yield and cost-effectiveness [13]. Genetech's method of production used chemically synthesized cDNA encoding for insulin A and B chains separately [14]. Thus, the two chains were purified and co-incubated under preferred conditions to accelerate generation growth of intact disulfide bond formation [15]. Alternatively, Eli Lilly used a single chemically synthesized cDNA encoding for human proinsulin with subsequent purification and excision of the C-peptide, yielding an active insulin product [15]. S. cerevisiae expression systems contain engineered insulin constructs with native A and B chains lacking the C-terminal B30 threonine, fused or linked by a small synthetic C-peptide [13]. The construct is made by the cDNA sequence fused with the alpha factor signal sequence in S. cerevisiae for expression of proinsulin [16]. This proinsulin product is purified and transformed to active insulin by a trypsin-mediated transpeptidation reaction in the presence of threonine ester [16]. Transgenic plants are used as expression systems due to their cost-effectiveness, high quality protein processing, absence of human pathogens and presence of eukaryotic machinery for post-translational modifications [17]. Recombinant human insulin has been produced in plant Arabidopsis thaliana via oil bodies [18]. Oil bodies are located inside oil seeds, which are comprised of a hydrophobic triacylglycerol core encapsulated by a phospholipid membrane and an outer wall of proteins known as oleosins [18-20]. The oil seeds are genetically engineered with the recombinant protein targeting oil bodies as oleosin fusion [18-20]. Further processing involves separating oil bodies via liquid-liquid phase separation to reduce chromatography steps in the purification of insulin [13]. Thereafter, the oil body is purified, then recombinant insulin is cleaved from the oleosin fusion partner and matured by trypsin digestion yielding an active insulin product. An alternative approach includes transforming tobacco and lettuce chloroplasts with human proinsulin made up of A, B and C chains fused with cholera toxin B subunit [21]. Production can yield up to $47 \%$ of proinsulin in lettuce leaves while proinsulin from tobacco leaves has been extracted with $98 \%$ purity [13]. The high level of biologically active proinsulin being expressed in transgenic plants provides low-cost production of injectable and oral proinsulin delivery [13].

\section{Advance in insulin application}

Insulin is primarily administered subcutaneously via vials and syringes [22]. However, due to limitations of using a vial or syringe for injection, development of insulin pens began to rise. Insulin pens are reusable, increase patient compliance, and are more accurate $[23,24]$. Advanced insulin pens include smart technology that keeps track of the last 16 doses for the patient through built-in calculators [25]. Injectable insulin still has drawbacks, leading researchers to take novel approaches to deliver insulin. Inhaled insulin products are currently being reviewed by the FDA for approval, as pulmonary routes offer advantages. Inhaled human insulin are of rDNA origin and utilize Technosphere drug delivery to reach the lungs [26]. Details of this pulmonary insulin delivery includes breath-activated inhalers containing pre-metered unit doses of insulin. Once this encounters the neutral $\mathrm{pH}$ of the alveolar epithelium it dissipates into liquid form [26]. An advantage seen here is the absence of gastrointestinal peptidases that break down insulin in the GI tract, subsequently bypassing the first-pass metabolism system [27]. Transdermal administration of insulin is a newer approach used where iontophoresis, sonophereis or phonophereis techniques are used [28,29]. In contrast to insulin delivery, stem cell therapy is being researched as an option to reverse insulin resistance. Stem cells have the capacity to differentiate into insulin-producing cells (IPCs), improve pancreatic regeneration and ameliorate insulin resistance - offering an alternative to islet cell transplant [30]. Specifically, mesenchymal stem cells (MSCs) have gained notoriety due to their ability to regenerate pancreatic islet $\beta$ cells, protect them from apoptosis and ameliorate insulin resistance of peripheral tissues by creating an optimal environment by secretion of paracrine factors [31-36]. Molecularly, MSCs differentiate into IPCs by reprogramming key transcription factors such as Pdx-1, Ngn-3, NeuroD1, Pax4 and Pax6 located in the endocrine section of the pancreas [37]. Several experiments have been conducted to demonstrate the efficacy of using MSCs, with Moriscot et al. being the first to induce human 
bone marrow MSCs (BM-MSC) differentiation into IPCs [38,39]. Comparative studies successfully differentiated Wharton's jelly-derived MSC (WJ-MSC) into mature $\beta$-cell phenotypes [40]. MSCs ability to promote regeneration of endogenous pancreatic islet $\beta$ cell via secretion of cytokines and growth factors that have paracrine and autocrine activities [41]. Lee et al. observed MSCs migrating toward islet of streptozocin (STZ)-induced diabetic mice where MSCs accelerated tissue repair by allowing endogenous cells to proliferate and regain their normal function [42]. These alternative insulin applications have led to new insulin products on the market.

\section{Recent advances in insulin patents \& future}

Many novel methods have been patented to enhance the delivery of insulin. Because oral insulin delivery is subject to enzymatic degradation, scientists have looked to nanoparticle-based approaches. This approach improves the bioavailability by protecting insulin from gastrointestinal conditions and enhancing the permeability of the enzyme. This is done by cellular uptake of nanoparticles or paracellular transport across tight junctions. One example of a current nanoparticle patent is an anionic natural polymer with medium chain fatty acids which are absorbed through the intestinal cell wall. The polymer blocks the release of insulin into the stomach to prevent degradation of the enzyme and is capable of opening tight junctions to enhance insulin absorption paracellularly [43-45].

In addition to the use of nanoparticles as a method of insulin delivery, other systems, such as the bioresponsive insulin deliver system, have been patented. This system comprises an artificial beta cell with a glucose-sensitive hydrogel membrane for the feedback-controlled delivery of insulin. This membrane traps glucose-oxidase enzymes in a hydrogel polymer, reduces the $\mathrm{pH}$ of the membrane and increases the permeability of the hydrogel membrane to insulin. Thus the system works to accelerate the release of insulin with increasing levels of glucose [43,46].

More recently, researchers have explored the use of liposomes, biliosomes and proliposomes for insulin delivery. These work by encapsulating insulin using the appropriate phospholipid/cholesterol ratio to prevent the leakage of insulin from the liposome core and degradation by enzymes. Bilosomes incorporate bile salts to the liposomes to stabilize the bilosome against bile salt degradation in the GI tract and enhance the fluidity of the membrane. Proliposomes are formed by film dispersion-freeze drying to form dried and free-flowed particles. These methods incorporate the use of liposomes to enhance gastrointestinal absorption and oral bioavailability of insulin [47].

While these are just a few examples of recent advances in insulin delivery patents, many other products exist. Some other examples include the incorporation of insulin into a water-in-oil emulsion by high-pressure homogenization to protect insulin against gastric degradation, encapsulating insulin in a small soft gelatin capsule and coating with a polyacrylic polymer to protect from enzymatic breakdown, and many more products which help prevent the degradation of insulin by the harsh gastrointestinal environment $[43,48]$.

\section{Conclusion}

Recent advances in production of insulin via MSCs have shown to be promising. Results from testing the efficacy of MSC use in animals have provided advantages, but some disadvantages still exist. Advantages include the ability of MSCs to manage hyperglycemic episodes by differentiating into IPCs, pancreatic regeneration and ameliorating insulin resistance in animal models [28]. Animals are not replicas of human patients with Type 2 diabetes mellitus, but they provide similar mechanisms of actions for MSCs. In addition to MSC insulin applications, new production systems have provided excellent benefits for patients with diabetes mellitus. Transgenic plant-based expression systems provide high-capacity production of insulin with low cost of production. This advantage will be beneficial as the number of diabetic patients continue to grow.

\section{Financial \& competing interests disclosure}

The authors have no relevant affiliations or financial involvement with any organization or entity with a financial interest in or financial conflict with the subject matter or materials discussed in the manuscript. This includes employment, consultancies, honoraria, stock ownership or options, expert testimony, grants or patents received or pending, or royalties.

No writing assistance was utilized in the production of this manuscript.

\section{References}

1. WHO Model List of Essential Medicines (2015). https://www.who.int/medicines/publications/essentialmedicines/en/

2. Zion Market Research. Human Insulin Market All Geared Up For Rapid Development With Expected Growth Of USD 43.6 Billion By 2021. zionmarketresearch.com/news/global-human-insulin-market 
3. Ahmad K. Insulin sources and types: a review of insulin in terms of its mode on diabetes mellitus. J. Tradit. Chin. Med. 34(2), 234-237 (2014).

4. Schwartz TW. The processing of peptide precursors. In: Molecular Biology of the Islets of Langerhans. Okamoto H. (Ed.) Cambridge University Press, 153-205 (1990).

5. Docherty K, Steiner DF. Post-translational proteolysis in polypeptide hormone biosynthesis. Annu. Rev. Physiol. 44, 625-638 (1982).

6. Seidah NG, Chrétien M. Proprotein and prohormone convertases of the subtilisin family recent developments and future perspectives. Trends Endocrinol. Metab. 3(4), 133-140 (1992).

7. Chance RE, Ellis RM, Bromer WW. Porcine proinsulin: characterization and amino acid sequence. Science 161(3837), 165-167 (1968).

8. Cutfield JF, Cutfield SM, Dodson EJ, Dodson GG, Emdin SF, Reynolds CD. Structure and biological activity of hagfish insulin. J. Mol. Biol. 132(1), 85-100 (1979).

9. Bajaj M, Blundell TL, Pitts JE et al. Dogfish insulin. Eur. J. Biochem. 135(3), 535-542 (1983).

10. Pollock HG, Kimmel JR, Hamilton JW et al. Isolation and structures of alligator gar (lepisosteus spatula) insulin and pancreatic polypeptide. Gen. Comp. Endocrinol. 67(3), 375-382 (1987).

11. Howey DC, Bowsher RR, Brunelle RL, Woodworth JR. Lys (B28), Pro (B29). human insulin: a rapidly absorbed analogue of human insulin. Diabetes 43(3), 396-402 (1994).

12. Mudaliar SR, Lindberg FA, Joyce $M$ et al. Insulin aspart (B28 asp-insulin): a fast-acting analog of human insulin: absorption kinetics and action profile compared with regular human insulin in healthy nondiabetic subjects. Diabetes Care 22(9), 1501-1506 (1999).

13. Baeshen NA, Baeshen MN, Sheikh A et al. Cell factories for insulin production. Microbial Cell Factories 13, 141 (2014).

14. Chance R, Frank B. Research, development production and safety of biosynthetic human insulin. Diabetes Care 16(3), 133-142 (1993).

15. Chance R, Glazer N, Wishner K. Insulin Lispro (Humalog) In: Biopharmaceuticals, an Industrial Perspective. Walsh G, Murphy B. (Eds). Springer, 149-172 (1999).

16. Kjeldsen T. Yeast secretory expression of insulin precursors. Appl. Microbiol. Biotechnol. 54, 277-286 (2000).

17. Staub JM, Garcia B, Graves J et al. High-yield production of a human therapeutic protein in tobacco chloroplasts. Nat. Biotechnol. 18 , 333-338 (2000).

18. Nykiforuk CL, Boothe JG, Murray EW et al. Transgenic expression and recovery of biologically active recombinant human insulin from Arabidopsis thaliana seeds. Plant Biotech. J. 4, 77-85 (2006).

19. Van Rooijen GJH, Moloney MM. Plant seed oil-bodies as carriers for foreign proteins. BioTechnology 13, 72-77 (1995).

20. Deckers H, Moloney MM, Baum A. The case for recombinant production of pharmaceutical proteins in plants. Annu. Rep. Med. Chem. 34, 237-245 (1999).

21. Boyhan D, Daniell H. Low-cost production of proinsulin in tobacco and lettuce chloroplasts for injectable or oral delivery of functional insulin and C-peptide. Plant Biotech. J. 9, 585-598 (2011).

22. Shah RB, Patel M, Maahs DM, Shah VN. Insulin delivery methods: past, present and future. Int. J. Pharm. Investig. 6(1), 1-9 (2016).

23. Selam JL. Evolution of diabetes insulin delivery devices. J. Diabetes Sci. Technol. 4, 505-513 (2010).

24. Penfornis A, Personeni E, Borot S. Evolution of devices in diabetes management. Diabetes Technol. Ther. 13(Suppl. 1), S93-S102 (2011).

25. Ignaut DA, Venekamp WJ. HumaPen memoir: a novel insulin-injecting pen with a dose-memory feature. Expert. Rev. Med. Devices 4 , 793-802 (2007).

26. Sorli C. New developments in insulin therapy for Type 2 diabetes. Am. J. Med. Stud. 127(10), 39-48 (2014).

27. Heinemann L. Alternative delivery routes: inhaled insulin. Diabetes Nutr. Metab. 15, 417-422 (2002).

28. Kanikkannan N. Iontophoresis-based transdermal delivery systems. BioDrugs. 16, 339-347 (2002).

29. Rao R, Nanda S. Sonophoresis: recent advancements and future trends. J. Pharm. Pharmacol. 61, 689-705 (2009).

30. Zang L, Hao H, Liu J, Li Y, Han W, Mu Y. Mesenchymal stem cell therapy in type 2 diabetes mellitus. Diabetol Metab. Syndr. 9,36 (2017).

31. Caplan AI, Dennis JE. Mesenchymal stem cells as trophic mediators. J. Cell Biochem. 98(5), 1076-1084 (2006).

32. Si Y, Zhao Y, Hao H et al. Infusion of mesenchymal stem cells ameliorates hyperglycemia in type 2 diabetic rats: identification of a novel role in improving insulin sensitivity. Diabetes 61(6), 1616-1625 (2012).

33. Hao H, Liu J, Shen J et al. Multiple intravenous infusions of bone marrow mesenchymal stem cells reverse hyperglycemia in experimental Type 2 diabetes rats. Biochem. Biophys. Res. Commun. 436(3), 418-423 (2013).

34. Gao X, Song L, Shen K et al. Bone marrow mesenchymal stem cells promote the repair of islets from diabetic mice through paracrine actions. Mol. Cell Endocrinol. 388(1-2), 41-50 (2014).

35. Lee RH, Seo MJ, Reger RL et al. Multipotent stromal cells from human marrow home to and promote repair of pancreatic islets and renal glomeruli in diabetic NOD/scid mice. Proc. Natl Acad. Sci. USA 103(46), 17438-17443 (2006). 
36. Chen L, Tredget EE, Wu PY, Wu Y. Paracrine factors of mesenchymal stem cells recruit macrophages and endothelial lineage cells and enhance wound healing. PLoS ONE 3(4), e1886 (2008).

37. Guney MA, Gannon M. Pancreas cell fate. Birth Defects Res. Part C Embryo Today Rev. 87(3), 232-248 (2009).

38. Moriscot C, de Fraipont F, Richard MJ et al. Human bone marrow mesenchymal stem cells can express insulin and key transcription factors of the endocrine pancreas developmental pathway upon genetic and/or microenvironmental manipulation in vitro. Stem Cells 23(4), 594-603 (2005).

39. Xie QP, Huang H, Xu B et al. Human bone marrow mesenchymal stem cells differentiate into insulin-producing cells upon microenvironmental manipulation in vitro. Differentiation 77(5), 483-491 (2009).

40. Wu LF, Wang NN, Liu YS, Wei X. Differentiation of Wharton's jelly primitive stromal cells into insulin-producing cells in comparison with bone marrow mesenchymal stem cells. Tissue Eng. Part A 15(10), 2865-2873 (2009).

41. Caplan AI, Dennis JE. Mesenchymal stem cells as trophic mediators. J. Cell. Biochem. 98(5), 1076-1084 (2006).

42. Lee RH, Seo MJ, Reger RL et al. Multipotent stromal cells from human marrow home to and promote repair of pancreatic islets and renal glomeruli in diabetic NOD/scid mice. Proc. Natl Acad. Sci. USA 103(46), 17438-17443 (2006).

43. Ramesan RM, Sharma CP. Recent advances in the oral delivery of insulin. Recent Pat. Drug Deliv. Formul. 8(2), 155-159 (2014).

44. Sawant KK, Dodiya SS. Recent advances and patents on solid lipid nanoparticles. Recent Pat. Drug Deliv. Formul. 2(2), 120-135 (2008).

45. Alai MS, Lin WJ, Pingale SS. Application of polymeric nanoparticles and micelles in insulin oral delivery. J. Food Drug Anal. 23(3), 351-358 (2015).

46. Trehan A, Ali A. Recent approaches in insulin delivery. Drug Develop. Industrial Pharm. 24(7), 589-597 (1998).

47. Wong C, Al-Salami H, Dass C. Recent advancements in oral administration of insulin-loaded liposomal drug delivery systems for diabetes mellitus. Int. J. Pharm. 549, 201-217 (2018).

48. Rekha MR, Sharma CP. Oral delivery of therapeutic protein/peptide for diabetes-future perspectives. Int. J. Pharm. 440(1), 48-62 (2013). 
